(C) 2019. This manuscript version is made available under the CC-BY-NC-ND 4.0

license http://creativecommons.org/licenses/by-nc-nd/4.0/

https://doi.org/10.1016/j.snb.2019.126954

\title{
Extracellular matrix protein microarray-based biosensor with single cell resolution: integrin profiling and characterization of cell-biomaterial interactions
}

Ainhoa Gonzalez-Pujana ${ }^{a, b^{*}}$, Edorta Santos-Vizcaino ${ }^{a, b^{*}}$, Maite García-Hernandoc,d, Beatriz Hernaez-Estradaa, Marian M. de Pancorbo ${ }^{d}$, Fernando Benito-Lopez ${ }^{c}$, Manoli Igartua a,b, Lourdes

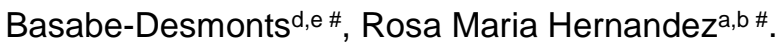

a NanoBioCel Group, Laboratory of Pharmaceutics, School of Pharmacy, University of the Basque Country, UPV/EHU, Vitoria-Gasteiz, Spain.

${ }^{b}$ Biomedical Research Networking Centre in Bioengineering, Biomaterials and Nanomedicine (CIBER-BBN). VitoriaGasteiz, Spain.

${ }^{c}$ Analytical Microsystems \& Materials for Lab-on-a-Chip (AMMa-LOAC) Group, Microfluidics Cluster UPV/EHU, Analytical Chemistry Department, University of the Basque Country UPV/EHU, Vitoria-Gasteiz, Spain

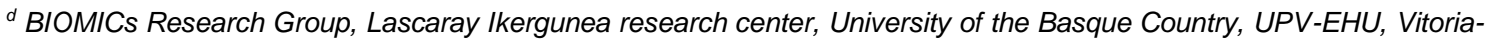
Gasteiz, Spain

elkerbasque, Basque Foundation for Science, Bilbao, Spain

A. Gonzalez-Pujana: ainhoa.gonzalez@ehu.eus, +34 945014518, orcid.org/0000-0001-6179-8237

E. Santos-Vizcaino: edorta.santos@ehu.eus, +34 945013093, orcid.org/0000-0001-7064-4563

M. García-Hernando: maite.garciah@ehu.eus, +34 945014034, orcid.org/0000-0001-8810-2746

B. Hernaez-Estrada: beatriz.hernaez@ehu.eus,+34 945014518.

M. M. de Pancorbo: marian.mdepancorbo@ehu.eus, +34945014528, orcid.org/0000-0002-7408-6068

F. Benito-Lopez: fernando.benito@ehu.eus,+34 945013045, orcid.org/0000-0003-0699-5507

M. Igartua: manoli.igartua@ehu.eus, +34 945013875, orcid.org/0000-0002-6439-4569

L. Basabe-Desmonts: lourdes.basabe@ehu.eus, +34 945014538, orcid.org/0000-0002-6638-7370

R.M. Hernandez: rosa.hernandez@ehu.eus, +34 945013095, orcid.org/0000-0002-3947-409X

\#Corresponding authors at:

Laboratory of Pharmaceutics, School of Pharmacy, University of the Basque Country (UPV/EHU); Paseo de la Universidad 7, 01006, Vitoria-Gasteiz, Spain. rosa.hernandez@ehu.eus.

Lascaray Research Center, University of the Basque Country (UPV/EHU); Avenida Miguel de Unamuno 3, 01007, Vitoria-Gasteiz, Spain. lourdes.basaba@ehu.eus.

*Authors contributed equally to this work. 


\section{ABSTRACT}

In the search of biomaterials that promote cell adhesion, it is crucial to explore the integrinsubstrate dynamic interactions given in a certain cell type to design successful biofunctionalization strategies. Here, we use a microarray platform for a thorough characterization of cell adhesion to a particular substrate. A biosensor based on an array of $20 \mu \mathrm{m}$ fibronectin circular isles was adapted to tissue culture treated plates to facilitate the performance of cell adhesion assays and the posterior affinity analyses. This sensitive analytical tool enables not only the evaluation of the cell adhesion kinetics, but also the integrin profiling and their contribution to cell attachment and adhesion strengthening via clustering. In particular, the biosensor was able to detect a significantly slower adhesion kinetics in fibroblasts, namely Baby Hamster Kidney Fibroblasts (BHK) and Human Dermal Fibroblasts (hDF), in comparison to other cell types such as $\mathrm{C}_{2} \mathrm{C}_{12}$ Mouse Myoblasts $\left(\mathrm{C}_{2} \mathrm{C}_{12}\right)$ or Human Mesenchymal Stem Cells (hMSCa). When directly comparing hDF and hMSCa, the analysis determined that the differing kinetics were caused by a distinct integrin expression profile. Whereas $\beta_{1}$-presenting integrins were the major responsible for hDF attachment, hMSCa adherence was importantly dependent on $\beta_{1}$ but also on other integrin classes. Additionally, results revealed that concerning cell adhesion consolidation, in hMSCa, both $\alpha_{v} \beta_{3}$ and $\beta_{1}$-subunit-presenting integrins contributed similarly; whereas in hDF, the latter played a more important role. Hence, our biosensor provided crucial information for the development of new cell-adhesive biomaterials, which are key in multiple biomedical fields including cell therapy or tissue engineering.

Keywords: Integrin; ECM; Cell therapy; Biosensor; Biomaterial; Single cell array. 


\section{INTRODUCTION}

In their natural niche, cells are surrounded by the extracellular matrix (ECM), a complex threedimensional (3D) network consisting of proteoglycans, glycosaminoglycans, glycoproteins and fibrous-forming proteins such as fibronectin, collagen or elastin [1]. Cells interact with their microenvironment through integrins, cell adhesion receptors that recognize ECM ligands. Integrins are heterodimers composed of an $\alpha$-subunit and a $\beta$-subunit. At least eighteen $\alpha$ and eight $\beta$ subunits have been identified in humans, and the different combinations give rise to 24 different integrin classes [2]. Their role is vital, since cell adhesion is a paramount feature for a correct cell function. Indeed, integrin-ECM binding results in the transduction of mechanical and biochemical signals that regulate diverse cellular processes such as cell adhesion, migration, proliferation and differentiation [3].

For that reason, over the last decades, special interest has been paid to engineering 3D matrices that mimic the physical, mechanical and biological properties of the ECM. Numerous biomaterials have demonstrated suitability for 3D culture of cells with multiple applications, including regenerative medicine [4], controlled drug delivery [5] or stem cell differentiation [6]. For instance, hydrogels made of natural polymers such as alginate [7] have been widely explored because of their versatility, enabling the modification of mechanical and degradation properties $[8,9]$. Moreover, these matrices offer the possibility to attach peptides naturally present in the ECM, thereby allowing immobilized cells to interact with otherwise inert biomaterials. This biofunctionalization, key for cell adhesion, has also been employed in other materials, such as titanium medical implants, promoting their interaction with host cells and accelerating the bone healing process [10].

Therefore, in the search of adequate materials for different biomedical applications, the focus is on surface modification [11]. It is possible to incorporate full-length ECM proteins, such as collagen, fibronectin, or gelatin. However, their use may be limited due to their heterogeneity, low protein stability and immunogenicity [12]. Moreover, the conformation and orientation of the protein hinder its correct presentation to the cells. An alternative that overcomes these limitations is the use of minimal cell recognizable sequences (short peptides responsible for cell adhesion) instead of the whole protein. Additional advantages comprise the more cost-effective synthesis 
and the easier characterization of peptides. Furthermore, because of their smaller size, it is possible to decorate the material surface with a higher density of cell binding domains [13]. The most widely employed short-peptide motif is Arginine-Glycine-Aspartate (RGD), a tri-peptide present in multiple ECM proteins such as fibronectin, vitronectin or laminin [14,15]. Its broad use is, in part, due to the fact that approximately half of the 24 integrins bind to ECM molecules in a RGD dependent manner. In fact, RGD peptides have been proven to stimulate cell adhesion on various materials, comprising polymers (synthetic or natural) and inorganic materials [16]. Some other relevant ECM moieties have also demonstrated cell attachment properties, either in combination with RGD or independently. Some examples are the sequences PHSRN, PRARI, YIGSR or IKVAV $[16,17]$ (Abbreviations are described in Table S1).

Despite the fact that numerous studies highlight the potential of matrices with mechano-adhesive properties, the affinity of each integrin class for these ECM cues is different. Therefore, for the design of effective functionalization strategies, it is necessary to determine the integrin classes that contribute to cell adhesion in the particular cell type employed. To date, it is possible to study the integrin expression via complex techniques such as flow cytometry; however, simple methods that evaluate not only the expression, but also the contribution of integrins to the whole adhesion process, from the initiation of cell attachment to the adhesion consolidation via integrin clustering, are still required.

In this regard, electrical impedance spectroscopy has been used to develop instruments to measure cell-substrate interactions and several equipment are commercially available [18]. However, these systems require complex fabrication techniques and they give an indirect measurement of cell adhesion and detachment based on electrical impedance, which can cause many artifacts [19]. Other optical [20,21] and piezoelectric [22] sensors have also successfully measured cell attachment and adhesion kinetics, but mainly for drug screening applications and not focused on integrin profiling.

On the other hand, sensor arrays represent an attractive analytical tool for exploring cell-ECM interactions [23] by optical means. Protein dot microarrays are fabricated by immobilizing the protein of interest onto a surface, commonly glass or silicon [24]. The rest of the surface is blocked with molecules that do not promote cell adhesion, such as bovine serum albumin (BSA) 
or nonfat milk blocking buffers, making every printed spot an independent experimental replicate. Numerous studies have taken advantage of these arrays to investigate how cell adhesion to printed ECM proteins regulate different cellular processes. These include the influence of cell spreading and shape in cell migration [25], cell-cell adhesion [26] or cell differentiation [27,28]. Additionally, arrays designed to hold a single cell per dot have been used to monitor adhesion kinetics of blood platelets to biofunctionalized substrates [29] as well as to measure platelet affinity to different ECM proteins [30] or to monitor the effect of antiplatelet drugs [31] by optical means. This platform enables a simple and accurate quantification of cell adhesion using a binary counting system of occupied and empty adhesion dots. The measurement is expressed by means of the Dot Array Occupancy (DAO, percentage of occupied dots).

Following this path, and focused on gathering fundamental information directly applicable to the development of new materials suited to the specific requirements of multiple biomedical applications, we developed a sensitive FN dot array biosensor, with single cell resolution, to explore the integrin profile and characterize the cell-biomaterial dynamic interactions.

\section{MATERIALS AND METHODS}

\subsection{Polydimethylsiloxane (PDMS) stamp fabrication}

A patterned stamp master wafer was fabricated by means of photolithography. The pattern design consisted of different regions. Each of these areas presented circular spots of a determined size $(20,50$ or $100 \mu \mathrm{m})$ separated from each other by $50 \mu \mathrm{m}$. To obtain PDMS stamps, a 10:1 (v/v) mixture of Sylgard 184 Silicon Elastomer and curing agent (Sigma Aldrich, Cat. No.: 761036-5EA) was poured over the stamp master and was degassed under vacuum for $30 \mathrm{~min}$. The mixture was cured $1 \mathrm{~h}$ at $70{ }^{\circ} \mathrm{C}$, carefully demolded and left in the oven for an additional hour to ensure complete curing.

\subsection{Preparation of $F N$-patterned surfaces}

The process was conducted at room temperature and $50-60 \%$ of humidity. Tissue culture plates (TCP) (Sigma-Aldrich, Cat. No.: CLS3513-50EA) were employed as substrates. To improve the protein transference to the surface, the plates were treated with oxygen plasma by being introduced in a plasma reactor (Harrick Plasma cleaner, PDC-002) for $40 \mathrm{~s}$ at an oxygen pressure 
of 0.7 bar and a high power. PDMS stamps were washed with $70 \%$ ethanol and dried prior to use. Subsequently, they were inked with $50 \mu \mathrm{L}$ of a phosphate buffered saline (PBS) solution containing a mixture of $50 \mu \mathrm{g} \mathrm{mL}^{-1}$ of bovine plasma FN (Thermo Fisher Scientific, Cat. No.: 33010-018,) and $6.25 \mu \mathrm{g} \mathrm{mL}^{-1}$ of carboxytetramethylrhodamine (TAMRA) (Thermo Fisher, Cat. No.: A23016) labeled BSA. After 30 min, the excess ink solution was removed from the PDMS surface with a pipette. Subsequently, stamps were washed with $\mathrm{ddH}_{2} \mathrm{O}$ and dried under a stream of nitrogen. Stamps were then placed in a plate well and incubated in contact with the substrate for additional $30 \mathrm{~min}$. After removing the PDMS stamps from the plate, $1 \mathrm{~mL}$ of a solution of $1 \%$ BSA in PBS was added to each micro-patterned well. Plates were incubated for $1 \mathrm{~h}$ at $4{ }^{\circ} \mathrm{C}$ with this solution to block any uncovered region of the surface. The plate wells were then rinsed with PBS and stored at $4{ }^{\circ} \mathrm{C}$ in the dark. Typically, patterned surfaces were used within the next $24 \mathrm{~h}$ after fabrication. Verification of homogeneous printing was performed by fluorescence microscopy (Nikon TMS, Hampton, NH).

\subsection{Cell culture}

Four cell types with different characteristics were selected for testing the applicability of the platform. Cells were seeded in T-flasks and cultured in the corresponding medium for each cell type. Baby Hamster Kidney Fibroblasts (BHK) (ATCC®, Cat. No: CCL-10 ${ }^{\mathrm{TM}}$ ) and $\mathrm{C}_{2} \mathrm{C}_{12}$ Mouse Myoblasts $\left(\mathrm{C}_{2} \mathrm{C}_{12}\right)\left(\mathrm{ATCC} \AA\right.$, Cat. No: CRL-1772 $\left.{ }^{\mathrm{TM}}\right)$ were grown in Dulbecco's Modified Eagle's Medium (DMEM) (ATCC $\AA$, Cat. No: 30-2002 ${ }^{\mathrm{TM}}$ ) supplemented with $10 \%$ FBS and $1 \%$ antibiotic/antimycotic solution. Adipose-Derived Human Mesenchymal Stem Cells (hMSCa) (ATCC $\AA$, Cat. No: PCS-500-011) were cultured in Mesenchymal Stem Cell Basal Medium (ATCC $\AA$, Cat. No: PCS-500-030 тм ) supplemented with the Mesenchymal Stem Cell Growth Kit (ATCC $\AA$, Cat. No: PCS-500-040). Primary Human Dermal Fibroblasts (hDF) (ATCC $\AA$, Cat. No: PCS-201-012) were grown in Fibroblast Basal Medium (ATCC $\AA$, Cat. No: PCS-201-030 тм supplemented with the Fibroblast Growth kit (ATCC $\AA$, Cat. No: PCS-201-041). Cells were maintained at $37{ }^{\circ} \mathrm{C}$ in a $5 \% \mathrm{CO}_{2} / 95 \%$ air atmosphere and passaged according to the necessities of each cell type by means of using trypsin- ethylenediaminetetraacetic acid (EDTA) (ATCC®, Cat. No: PCS-999-003).

\subsection{Cell seeding and DAO determination}


For cell seeding in the substrate, $2 \times 10^{5}$ cells were suspended in $1 \mathrm{~mL}$ of the adequate fetal bovine serum (FBS)-free medium and added in each well. Plates were protected from light and placed in a rocker (Thermo Fisher, Cat. No.: M48720-33Q). Agitation was maintained in an incubator, at $37^{\circ} \mathrm{C}$ in a $5 \% \mathrm{CO}_{2}$ air atmosphere, for the time interval required for each experiment. Subsequently, the unattached cells were washed off and processed, following the requirements of the performed bioassay.

For adhesion quantification, fluorescence microscopy images were obtained by means of an inverted microscope (Nikon TMS, Hampton, NH). The 4X objective field of view was used to capture approximately 1000 dots of $20 \mu \mathrm{m}$ in each micrograph. For the same substrate zone, two images were acquired: one displaying the TAMRA-labeled pattern, and a brightfield image capturing the adhered cells. At least three different substrate areas were processed for each experimental point, gathering at least 3000 dots, by means of ImageJ software (Rasband, W.S., ImageJ, US National Institutes of Health, Bethesda, Rockville, MD, https://imagej. nih.gov/ij/, 1997-2016). In particular, micrographs were analyzed using the cell counter tool and the DAO was expressed as the mean value of the percentage of FN dots occupied with adhered cells.

\subsection{Integrin profiling assay}

For determining the influence of blocking specific integrins in cell adhesion, cells were pretreated with the adequate anti-integrin antibodies (Abcam, anti-Integrin $\beta_{1}$ antibody, Cat. No: ab24693, anti-Integrin $\alpha_{v} \beta_{3}$ antibody, Cat. No: ab78289) for $1 \mathrm{~h}$ at $37{ }^{\circ} \mathrm{C}$ in $5 \% \mathrm{CO}_{2}$ and agitation. Afterwards, cells were seeded in the patterned plates and incubated an additional hour as previously described. Once the unattached cells were washed off, cells were fixed and the DAO was quantified as detailed above.

\subsection{Immunostaining}

Cells were pretreated with anti-integrin antibodies and incubated in the substrates for $1 \mathrm{~h}$ at 37 ${ }^{\circ} \mathrm{C}$ in $5 \% \mathrm{CO}_{2}$ and agitation. After washing off the non-attached excess, cells were fixed, permeabilized and incubated with anti-phosphorylated focal adhesion kinase ( $p F A K$ ) primary antibody (Abcam, Anti-FAK phospho Y397, Cat. No: ab81298). Afterwards, samples were stained with the Alexa Fluor 488-labelled secondary antibody (Abcam, Cat. No: ab150077), Alexa Fluor 
594- labelled phalloidin for F-actin (Thermo Fisher Scientific, Cat. No: A12381) and DAPI for the nuclei (Thermo Fisher Scientific, Cat. No: D1306). Fluorescent micrographs were acquired by means of an inverted fluorescence microscope (Nikon TMS, Hampton, NH).

\subsection{Cell-substrate attachment strength determination}

After being pretreated with anti-integrin antibodies, cells were incubated in the substrates in agitation for $1 \mathrm{~h}$ at $37{ }^{\circ} \mathrm{C}$ in $5 \% \mathrm{CO}_{2}$. Once the non-adhered cells were washed, the DAO was quantified as previously described to obtain the initial occupancy values. Afterwards, a detachment assay was performed. Specifically, each micro-patterned well was subjected to 5 cycles of liquid rinse/aspiration, and the DAO was quantified again. The comparison between the pre-detachment and post-detachment DAO values determined the strength of the cell-FN binding.

\subsection{Flow cytometry}

The results obtained with our platform were validated by flow cytometry. A suspension of $10^{6}$ cells was dyed for 10 min at $4{ }^{\circ} \mathrm{C}$ in the dark with human CD29-PEVIO770 antibodies for $\beta_{1}$ detection (Miltenyi Biotec, Cat. No: 130-101-281) and human CD51/CD61-APC antibodies for $\alpha_{v} \beta_{3}$ determination (Miltenyi Biotec, Cat. No: 130-103-745) following the manufacturer's instructions. IgG1-APC (Miltenyi Biotec, Cat. No: 130-113-758) and IgG1-PE-vio770 (Miltenyi Biotec, No: $130-$ 113-764) antibodies were used as isotype controls. Cells were then washed and re-suspended in a buffer containing $0.5 \%$ BSA and 2 mM EDTA in PBS to be processed by flow cytometry (MACSQuant Analyzer, Miltenyi Biotec).

\subsection{Data analysis and statistics}

All samples were assayed at least in triplicate and results are shown as mean \pm SD for line and bar graphs. The normal distribution of the data was checked by the Shapiro-Wilk test. One sample t-test was employed to determine if data differed significantly from a determined mean value. To detect statistical significances between two groups, a two-tailed t-test was performed, while oneway ANOVA was used for multiple comparisons. For the latter, an additional test, the Levene test, was used to determine the homogeneity of variances. If homogeneous, the Bonferroni post-hoc was applied and if non-homogeneous, the Tamhane post-hoc was employed. In the case of non- 
normally distributed data, a Mann-Whitney non-parametric test was applied. All statistical computations were performed by means of SPSS 23 (IBM SPSS, Chicago, IL).

\section{RESULTS AND DISCUSSION}

\subsection{Platform optimization for single cell studies in $B H K, C_{2} C_{12}$, hMSCa and hDF cells}

In the development of a platform to explore cell adhesive properties, micro-contact printing was adapted to print FN dot arrays on TCP wells (Fig. 1A). A patterned stamp master was obtained by photolithography and used to fabricate PDMS stamps presenting circular pillars of various sizes. Indeed, the versatility of the technique enables the printing of protein isles of different sizes and shapes (Fig. S1). The stamps were incubated with a PBS solution containing FN and TAMRAlabeled BSA, to obtain a positive control for protein printing, and stamped in the TCP wells resulting in FN dot arrays. Fluorescence microscopy indicated a homogeneous printing of dots (Fig. 1B). To verify the uniformity of the pattern, we evaluated the CV of the dots' fluorescence intensity (Fig. 1C), size (Fig. 1D) and circularity (Fig. 1E), and obtained CV values of 6.45, 11.26 and $3.05 \%$, respectively ( $n=300$ dots per image field). The sample was therefore considered homogeneous, which permitted cells to adhere to the dots with equal probability, as observed throughout all the study. To confirm this observation, within the same sample, we calculated the CV of the dot array occupancy (DAO) of the replicates $(n=4)$. In particular, we evaluated samples from the different cell types employed in the study, and obtained low CV \% values as observed in Table S2.

Subsequently, we assayed the stability of the pattern by incubating a suspension of cells in both, a freshly fabricated substrate and an overnight $4{ }^{\circ} \mathrm{C}$ stored substrate. We obtained no significant differences in the FN dot array occupancy (DAO), confirming the overnight stability of the platform (Fig. 1F). This represents a technical advantage, since it is possible to fabricate and store substrates on one day and perform the cell studies on the following.

To demonstrate the real applicability of the biosensor to study different adhesion parameters, cells with differing characteristics were employed. Specifically, we chose established cell lines and primary cells with varying origin and morphology as shown in Fig. 2A. Moreover, because of their different sizes, it was necessary to determine the adequate dot diameter that resulted in a 
single cell adhesion for each cell type. Therefore, cells were incubated in substrates presenting dots of 20,50 and $100 \mu \mathrm{m}$ (Fig. 2B). For the bigger isles, 50 and $100 \mu \mathrm{m}$ dots, we did not obtain a single cell adhesion $(p<0.001)$ in any case. Multiple cells were attached to each of these dots, and interestingly, the number varied when using cell lines or primary cells. Specifically, the cell lines in this study, namely $\mathrm{BHK}$ and $\mathrm{C}_{2} \mathrm{C}_{12}$, presented a smaller size than primary hMSCa and hDF, and hence, a higher number of cells fitted each 50 or $100 \mu \mathrm{m}$ dot. Despite these differences, FN isles of $20 \mu \mathrm{m}$ provided a single cell adhesion pattern for all the four cells. Therefore, $20 \mu \mathrm{m}$ circular dots were printed for the rest of the study to carry out single cell assays.

\subsection{Cell type determined cell adhesion kinetics}

First, we studied cell adhesion kinetics following the procedure shown in Fig. $3 \mathrm{~A}$. BHK, $\mathrm{C}_{2} \mathrm{C}_{12}$, hMSCa and hDF cells were incubated in a FN dot array substrate for two hours and the DAO was determined at different time points. For every cell, we observed how the DAO increased over time until reaching a plateau (Fig. 3B). The adhesion curves were fitted to a first order kinetics, providing values for the rate constant $(k)$ and the $t_{50}$, defined as the time point at which the $50 \%$ of the dot arrays are occupied. With these parameters, we were able to identify two different kinetics. Presenting significantly higher $\mathrm{k}$ and lower $\mathrm{t}_{50}$ values, $\mathrm{C}_{2} \mathrm{C}_{12}$ and $\mathrm{hMSCa}$ adhered significantly faster to the substrate than BHK and hDF $(p<0.001)$ (Fig. $3 C)$.

Although it was possible to hypothesize that the kinetics might be importantly influenced for being primary cells or a cell line, our results indicated that the cell type was the major determinant. We observed that the two fibroblasts included in the study, BHK and hDF, presented similar kinetics in spite of the fact that the former is a mouse cell line and the latter has a human primary origin. Since cell-matrix interactions are mainly mediated by integrins [2], we hypothesized that the differences observed in the adhesion kinetics may be given by a distinct integrin expression profile in each cell type.

\section{3. $h M S C a$ and $h D F$ presented a different integrin expression profile}

Our next goal was to study the integrin contribution to cell attachment. For the following sets of experiments, we continued with two of the four cells. In particular, we selected hMSCa and hDF 
for presenting contrasting kinetics and for being the most attractive from a therapeutic standpoint because of their human and primary origin [32-34].

With that aim, we followed the procedure described in Fig. 4A. We first incubated the cells with anti-integrin antibodies, specifically blocking either the $\alpha_{v} \beta_{3}$ integrin, or all the integrins presenting the $\beta_{1}$ subunit. These integrins were selected for being primary FN binding receptors in both cell types $[28,35]$. Subsequently, cells were seeded in the FN dot array platform and the DAO was quantified. Regarding hMSCa (Fig. 4B), DAO was not significantly affected when blocking the $\alpha_{v} \beta_{3}$ integrin, but it was when blocking all the integrins presenting the $\beta_{1}$ subunit $(p<0.001)$. Similar results were obtained for hDF $(p<0.001)$ (Fig. 4C). However, in this case, a significantly greater impact of $\beta_{1}$ blocking was observed, as demonstrated when normalizing the data to directly compare the two cell types $(p<0.05)$ (Fig. 4D).

When blocking $\beta_{1}$ subunit presenting integrins, approximately a $40 \%$ of the hMSCa cells were able to attach to the substrate, but only around a $2 \%$ of hDF. This indicates a different integrin profile; while in hDF $\beta_{1}$ integrin expression is major, in $\mathrm{hMSCa}$, their influence is balanced up to a certain point by other integrins, including $a_{v}$-class integrins, which also play a role in cell attachment.

On the other hand, this assay proved the sensitivity of the platform, since the sensor was capable to discern differences in DAO when blocking a single integrin, $\alpha_{v} \beta_{3}$, versus blocking the $\beta_{1}$ domain, which is present in a number of integrins [36].

3.4. $\alpha_{v} \beta_{3}$-mediated crosstalk was crucial for integrin clustering and cell-substrate interaction strengthening

In order to study the contribution of each integrin class to cell adhesion consolidation via focal adhesion formation, we first performed an immunocytochemistry assay for focal adhesion kinase (FAK) staining. FAK is a non-receptor protein-tyrosine kinase that transforms the cell-external biomechanical stimuli into biochemical signals, activating important intracellular signaling pathways [37]. This occurs preferentially by the specific phosphorylation of FAK-internal tyrosine residues, being two of the most relevant the tyrosine residues Y397 and Y861 [38]. Therefore, we once again blocked the $\alpha_{v} \beta_{3}$ integrin or all the integrins presenting the $\beta_{1}$ subunit in hMSCa or 
hDF cells, and subsequently seeded them in the FN dot array substrate for $1 \mathrm{~h}$. Cells were fixed and stained for pFAK-Y397 or pFAK-Y861. Micrographs showed FAK-Y397 phosphorylation in all of the groups for both cell types (Fig. 5A). Similar results were obtained when staining for the tyrosine residue Y861 (Fig. S2). Hence, this commonly used technique failed to determine the actual contribution of each integrin to the achievement of a functional adhesion, and did not discriminate among blocking the $\alpha_{v} \beta_{3}$ integrin, or all the $\beta_{1}$ integrins.

On the contrary, a detachment assay performed in our biosensor was able to provide a deeper insight into cell-substrate interaction. $\alpha_{v} \beta_{3}$ or $\beta_{1}$-presenting integrins were blocked and cells incubated in the FN dot array. The DAO was quantified and subsequently, a detachment force was applied by liquid aspiration. After five cycles, the DAO was quantified again and compared to the initial determination. Micrographs showing adhered cells prior and after the application of the detachment force are shown in Fig. S3 and S4. Although we previously detected that $\alpha_{v} \beta_{3}$ blocking did not alter the DAO, the adhered cells in this group presented less resistance to detachment forces than the control group in both, hMSCa and hDF $(p<0.001)$ (Fig. 5B). This phenomenon may be explained by the crosstalk that exists between $\alpha_{v}$ and $\beta_{1}$ integrins. It has been reported that once engaged, $\alpha_{v}$ integrins induce $\alpha_{5} \beta_{1}$ integrin clustering, strengthening cell adhesion [39]. Therefore, the absence of $\alpha_{v}$ integrins would impede the crosstalk and as a result, weaken the cell-substrate interaction. Consequently, $\beta_{1}$ blocking also debilitated the cell binding, because of the absence of $\beta_{1}$-class integrin clusters which are responsible for providing a stronger attachment.

Despite both cell types showed this behavior, the $\beta_{1}$ blocking prevented cell adhesion strengthening more importantly than $\alpha_{v} \beta_{3}$ blocking in hDF ( $\left.p<0.05\right)$, but not in hMSCa (Fig. 5C). This suggests a greater impact of $\beta_{1}$ integrins in hDF in comparison to hMSCa, confirming the results previously obtained (Fig. 4D). Moreover, this finding corroborates previous data highlighting the vital role of $\alpha_{4} \beta_{1}$ and $\alpha_{5} \beta_{1}$ integrins in hDF cells [35].

\subsection{Validation of the method by means of flow cytometry}

To validate the potential of the platform as a simple and rapid tool to determine integrin contribution to the cell adhesion process, $\alpha_{v} \beta_{3}$ and $\beta_{1}$ expression was determined by flow cytometry. Regarding $\alpha_{v} \beta_{3}$ integrin (Fig. $6 \mathrm{~A}$ ), whereas a $99.97 \%$ of the hMSCa cells presented it, 
only a $51.62 \%$ of hDF expressed it above the detectable threshold (Fig. 6B). Consequently, when quantifying the mean fluorescence intensity (MFI), the expression of the integrin was significantly higher in hMSCa $(p<0.001)$ (Fig. 6B). Regarding $\beta_{1}$, (Fig. 6C) although for both, hMSCa and hDF, all the cells presented it, the determination of the MFI showed an importantly higher expression in hDF $(p<0.001)$ (Fig. 6D). Altogether, these findings highlight the differences between the two cell types, showing that hDF cells present a lower $\alpha_{v} \beta_{3}$ and a higher $\beta_{1}$ expression than hMSCa.

This data support the three main conclusions provided by our method. First, considering that $\alpha_{v}-$ class integrins initiate cell attachment by binding FN quicker than $\beta_{1}$-presenting integrins such as $\alpha_{5} \beta_{1}$ [35], the faster kinetics indicated a higher density of $\alpha_{v}$-class integrins in $C_{2} C_{12}$ and hMSCa than in BHK and hDF. Second, the integrin expression profile was different in hMSCa and hDF, being major the role of $\beta_{1}$-presenting integrins in $\mathrm{hDF}$, and important but not unique in hMSCa. Third, $\alpha_{v} \beta_{3}$ and $\beta_{1}$ contributed similarly to hMSCa adhesion strengthening, but for hDF, the role of $\beta_{1}$ integrins was still greater.

Although the integrins may be explored through flow cytometry, this technique is more complex and only provides information regarding the integrin expression, but not their actual contribution in the whole adhesion process. Moreover, it is important to note that for flow cytometry, cells are detached and studied in suspension. Hence, the biological processes dependent on cell adhesion, involving important phosphorylation pathways, are limited. Contrarily, our platform offered the possibility to study attached cells in an environment that mimics their natural niche and predict both, integrin expression profile and their role in functional adhesion formation via integrin crosstalk and clustering. This results in a valuable biosensor for the rapid determination of the adhesive properties of a specific cell type, generating crucial information directly applicable to the field of biomimetic material science.

Multiple integrins bind the tri-peptide RGD, which has been recognized as the essential cell adhesion site in FN and other natural components of the ECM [14,40]. However, the affinity for this motif varies among the different integrins. For instance, while both $\alpha_{v} \beta_{3}$ and $\alpha_{5} \beta_{1}$ integrins bind RGD, the latter also requires the PHSRN synergy site, present in natural FN in close proximity to RGD, to establish cell adhesion $[35,41,42]$. Likewise, $\alpha_{4} \beta_{1}$ integrins also bind a 
synergistic site, in this case the PRARI sequence [42]. Therefore, it is crucial to explore the contribution of integrins in a particular cell type in order to correctly design efficient biofunctionalization strategies [43].

By means of our biosensor, we were able to determine the contribution of $\alpha_{v} \beta_{3}$ and $\beta_{1}$ integrins in hDF and hMSCa cells, gathering fundamental information to optimize material biomimetization. In particular, our data indicate that although the inclusion of synergy motifs such as PHSRN or PRARI may be beneficial in hMSCa, the presence of the sole RGD may promote an important cell response. Contrarily, the accompaniment of RGD with the synergistic peptides in hDF may be essential, considering the smaller contribution of $\alpha_{\vee} \beta_{3}$ and the potent effects of $\beta_{1}$.

Generally, the design of biofunctionalized matrices goes through an optimization stage in which the material is modified with different ECM-derived peptides at different densities. Once all the materials are functionalized, complex 3D matrices are fabricated with each of the biomimetization alternatives and cells are cultured within them to determine their behavior. A preliminary screening in our biosensor would rapidly predict the best strategies to follow, importantly limiting the number of groups to test. Here, FN dot arrays were employed; however, it is possible to pattern other peptides or proteins of interest or even mixtures of different moieties that a priori may seem interesting [44]. This would not only save time but would be significantly more cost-effective, since the amount of peptide to be used for the screening is significantly lower than the required to modify materials and manufacture matrices with them. Moreover, the platform allows the study of any receptor class by only selecting the most suitable anti-integrin antibodies and can be easily performed in multiple cell types in parallel, resulting in a powerful and high throughput analytical tool.

\section{CONCLUSION}

In this study, we developed an ECM protein dot microarray biosensor for the characterization of cell adhesion to a particular substrate with single cell resolution. By exploring cell adhesion kinetics, integrin profile and integrin contribution to adhesion formation and consolidation, we were able to detect differences in the adhesive properties of various cell types, including primary and established cell lines from different sources. The sensor enables to study in depth the affinity of cells for different substrates, it is not limited to FN. This results in a useful tool for the design of 
biomimetic materials, which are key in multiple fields including cell therapy, tissue engineering or targeted drug delivery. The potential of this biosensor lies on its high sensitivity, accuracy, simplicity and low cost and will be of interest for the whole community of researchers interested in cell-substrate interactions.

\section{ACKNOWLEDGEMENTS}

Authors thank for the projects SAF2017-82292-R and BIO2016-80417-P (MINECO/AEI/FEDER, UE), ICTS "NANBIOSIS" (Drug Formulation Unit, U10) and the support from the Basque Country Government (Grupos Consolidados, No ref: IT907-16). A. Gonzalez-Pujana thanks the Basque Government (Department of Education, Universities and Research) for the PhD grant (PRE_2018_2_0133). M. Garcia-Hernando thanks the University of the Basque Country for the PhD grant (PIF16/204).

\section{REFERENCES}

[1] A.D. Theocharis, S.S. Skandalis, C. Gialeli, N.K. Karamanos, Extracellular matrix structure, Adv. Drug Deliv. Rev. 97 (2016) 4-27. https://doi.org/10.1016/j.addr.2015.11.001.

[2] Y. Takada, X. Ye, S. Simon, The integrins, Genome Biol. 8 (2007) 215-2007-8-5-215. https://doi.org/10.1186/gb2007-8-5-215.

[3] H.T. Aiyelabegan, E. Sadroddiny,Fundamentals of protein and cell interactions in biomaterials, Biomed. Pharmacother. 88 (2017) 956-970. https://doi.org/10.1016/j.biopha.2017.01.136.

[4] L. Wang, J. Huang, C. Huang, Q. Li, L. Liu, S. Luo, J. Xiao,Adult Stem Cells and Hydrogels for Cartilage Regeneration, Curr. Stem Cell. Res. Ther. 13 (2018) 533-546. https://doi.org/10.2174/1574888X12666170511142917.

[5] A.A. Tomei, C. Villa, C. Ricordi,Development of an encapsulated stem cell-based therapy for diabetes, Expert Opin. Biol. Ther. 15 (2015) 1321-1336. https://doi.org/10.1517/14712598.2015.1055242.

[6] Q. Huang, Y. Zou, M.C. Arno, S. Chen, T. Wang, J. Gao, A.P. Dove, J. Du,Hydrogel scaffolds for differentiation of adipose-derived stem cells, Chem. Soc. Rev. 46 (2017) 6255-6275. https://doi.org/10.1039/c6cs00052e.

[7] T. Andersen, P. Auk-Emblem, M. Dornish,3D Cell Culture in Alginate Hydrogels, Microarrays (Basel). 4 (2015) 133-161. https://doi.org/10.3390/microarrays4020133.

[8] K.H. Vining, A. Stafford, D.J. Mooney,Sequential modes of crosslinking tune viscoelasticity of cell-instructive hydrogels, Biomaterials. 188 (2019) 187-197. https://doi.org/10.1016/j.biomaterials.2018.10.013.

[9] A. Gonzalez-Pujana, A. Rementeria, F.J. Blanco, M. Igartua, J.L. Pedraz, E. Santos-Vizcaino, R.M. Hernandez, The role of osmolarity adjusting agents in the regulation of encapsulated cell behavior to provide a safer and more predictable delivery of therapeutics, Drug Deliv. 24 (2017) 1654-1666. https://doi.org/10.1080/10717544.2017.1391894. 
[10] M. Heller, V.V. Kumar, A. Pabst, J. Brieger, B. Al-Nawas, P.W. Kammerer,Osseous response on linear and cyclic RGD-peptides immobilized on titanium surfaces in vitro and in vivo, J. Biomed. Mater. Res. A. 106 (2018) 419-427. https://doi.org/10.1002/jbm.a.36255.

[11] A.L. Hook, D.G. Anderson, R. Langer, P. Williams, M.C. Davies, M.R. Alexander,High throughput methods applied in biomaterial development and discovery, Biomaterials. 31 (2010) 187-198. https://doi.org/10.1016/j.biomaterials.2009.09.037.

[12] K. Sivaraman, C. Shanthi,Matrikines for therapeutic and biomedical applications, Life Sci. 214 (2018) 22-33. https://doi.org/10.1016/j.Ifs.2018.10.056.

[13] E. Santos, A. Garate, J.L. Pedraz, G. Orive, R.M. Hernandez,The synergistic effects of the RGD density and the microenvironment on the behavior of encapsulated cells: in vitro and in vivo direct comparative study, J. Biomed. Mater. Res. A. 102 (2014) 3965-3972. https://doi.org/10.1002/jbm.a.35073.

[14] J. Takagi,Structural basis for ligand recognition by RGD (Arg-Gly-Asp)-dependent integrins, Biochem. Soc. Trans. 32 (2004) 403-406. https://doi.org/10.1042/BST0320403.

[15] A. Garate, E. Santos, J.L. Pedraz, R.M. Hernandez, G. Orive,Evaluation of different RGD ligand densities in the development of cell-based drug delivery systems, J. Drug Target. 23 (2015) 806-812. https://doi.org/10.3109/1061186X.2015.1020428.

[16] U. Hersel, C. Dahmen, H. Kessler,RGD modified polymers: biomaterials for stimulated cell adhesion and beyond, Biomaterials. 24 (2003) 4385-4415. https://doi.org/10.1016/S0142-9612(03)00343-0.

[17] A. Farrukh, F. Ortega, W. Fan, N. Marichal, J.I. Paez, B. Berninger, A.D. Campo, M.J. Salierno,Bifunctional Hydrogels Containing the Laminin Motif IKVAV Promote Neurogenesis, Stem Cell. Reports. 9 (2017) 1432-1440. https://doi.org/10.1016/j.stemcr.2017.09.002.

[18] ACEA Biosciences - xCELL Ligence System, Cell Index, E-Plate. https://www.aceabio.com/about/, Last accessed March 2019 (2014).

[19] A. Susloparova, D. Koppenhofer, J.K. Law, X.T. Vu, S. Ingebrandt,Electrical cell-substrate impedance sensing with field-effect transistors is able to unravel cellular adhesion and detachment processes on a single cell level, Lab. Chip. 15 (2015) 668-679. https://doi.org/10.1039/c4lc00593g.

[20] B. Peter, R. Ungai-Salanki, B. Szabo, A.G. Nagy, I. Szekacs, S. Bosze, R. Horvath,High-Resolution Adhesion Kinetics of EGCG-Exposed Tumor Cells on Biomimetic Interfaces: Comparative Monitoring of Cell Viability Using Label-Free Biosensor and Classic End-Point Assays, ACS Omega. 3 (2018) 3882-3891. https://doi.org/10.1021/acsomega.7b01902.

[21] I. Szekacs, N. Orgovan, B. Peter, B. Kovacs, R. Horvath,Receptor specific adhesion assay for the quantification of integrin-ligand interactions in intact cells using a microplate based, label-free optical biosensor, Sensors and Actuators B: Chemical. 256 (2018) 729-734. https://doi.org/10.1016/j.snb.2017.09.208.

[22] Z. Fohlerová, J. Turánek, P. Skládal,The cell adhesion and cytotoxicity effects of the derivate of vitamin $E$ compared for two cell lines using a piezoelectric biosensor, Sensors and Actuators B: Chemical. 174 (2012) 153157. https://doi.org/10.1016/j.snb.2012.08.047.

[23] B. Delalat, D.M. Rojas-Canales, S. Rasi Ghaemi, M. Waibel, F.J. Harding, D. Penko, C.J. Drogemuller, T. Loudovaris, P.T. Coates, N.H. Voelcker,A Combinatorial Protein Microarray for Probing Materials Interaction with Pancreatic Islet Cell Populations, Microarrays (Basel). 5 (2016). https://doi.org/10.3390/microarrays5030021.

[24] Y. Huang, H. Zhu,Protein Array-based Approaches for Biomarker Discovery in Cancer, Genomics Proteomics Bioinformatics. 15 (2017) 73-81. https://doi.org/10.1016/j.gpb.2017.03.001.

[25] P. Monzo, Y.K. Chong, C. Guetta-Terrier, A. Krishnasamy, S.R. Sathe, E.K. Yim, W.H. Ng, B.T. Ang, C. Tang, B. Ladoux, N.C. Gauthier, M.P. Sheetz,Mechanical confinement triggers glioma linear migration dependent on formin FHOD3, Mol. Biol. Cell. 27 (2016) 1246-1261. https://doi.org/10.1091/mbc.E15-08-0565.

[26] J.Y. Sim, J. Moeller, K.C. Hart, D. Ramallo, V. Vogel, A.R. Dunn, W.J. Nelson, B.L. Pruitt,Spatial distribution of cell-cell and cell-ECM adhesions regulates force balance while main-taining E-cadherin molecular tension in cell pairs, Mol. Biol. Cell. 26 (2015) 2456-2465. https://doi.org/10.1091/mbc.E14-12-1618. 
[27] R.M. Duffy, Y. Sun, A.W. Feinberg,Understanding the Role of ECM Protein Composition and Geometric Micropatterning for Engineering Human Skeletal Muscle, Ann. Biomed. Eng. 44 (2016) 2076-2089. https://doi.org/10.1007/s10439-016-1592-8.

[28] J. Lee, A.A. Abdeen, X. Tang, T.A. Saif, K.A. Kilian,Geometric guidance of integrin mediated traction stress $\begin{array}{llllll}\text { during stem cell } & \text { differentiation, } & \text { Biomaterials. } & 69 & \text { (2015) }\end{array}$ https://doi.org/10.1016/j.biomaterials.2015.08.005.

[29] A. Lopez-Alonso, B. Jose, M. Somers, K. Egan, D.P. Foley, A.J. Ricco, S. Ramstrom, L. Basabe-Desmonts, D. Kenny, Individual platelet adhesion assay: measuring platelet function and antiplatelet therapies in whole blood via digital quantification of cell adhesion, Anal. Chem. 85 (2013) 6497-6504. https://doi.org/10.1021/ac401076s.

[30] L. Basabe-Desmonts, S. Ramstrom, G. Meade, S. O'Neill, A. Riaz, L.P. Lee, A.J. Ricco, D. Kenny,Single-step separation of platelets from whole blood coupled with digital quantification by interfacial platelet cytometry (iPC), Langmuir. 26 (2010) 14700-14706. https://doi.org/10.1021/la9039682.

[31] B. Jose, P. McCluskey, N. Gilmartin, M. Somers, D. Kenny, A.J. Ricco, N.J. Kent, L. Basabe-Desmonts,SelfPowered Microfluidic Device for Rapid Assay of Antiplatelet Drugs, Langmuir. 32 (2016) 2820-2828. https://doi.org/10.1021/acs.langmuir.5b03540.

[32] V. Thulabandu, D. Chen, R.P. Atit,Dermal fibroblast in cutaneous development and healing, Wiley Interdiscip. Rev. Dev. Biol. 7 (2018) 10.1002/wdev.307. Epub 2017 Dec 15. https://doi.org/10.1002/wdev.307.

[33] Y. Wang, X. Chen, W. Cao, Y. Shi,Plasticity of mesenchymal stem cells in immunomodulation: pathological and therapeutic implications, Nat. Immunol. 15 (2014) 1009-1016. https://doi.org/10.1038/ni.3002.

[34] A. Mohr, R. Zwacka,The future of mesenchymal stem cell-based therapeutic approaches for cancer - From cells to ghosts, Cancer Lett. 414 (2018) 239-249. https://doi.org/10.1016/j.canlet.2017.11.025.

[35] K. Hozumi, K. Nakamura, H. Hori, M. Miyagi, R. Nagao, K. Takasaki, F. Katagiri, Y. Kikkawa, M. Nomizu,Mixed Fibronectin-Derived Peptides Conjugated to a Chitosan Matrix Effectively Promotes Biological Activities through Integrins, alpha4beta1, alpha5beta1, alphavbeta3, and Syndecan, Biores Open Access. 5 (2016) 356-366. https://doi.org/10.1089/biores.2016.0037.

[36] D. Cox, M. Brennan, N. Moran,Integrins as therapeutic targets: lessons and opportunities, Nat. Rev. Drug Discov. 9 (2010) 804-820. https://doi.org/10.1038/nrd3266.

[37] P. Tomakidi, S. Schulz, S. Proksch, W. Weber, T. Steinberg,Focal adhesion kinase (FAK) perspectives in mechanobiology: implications for cell behaviour, Cell Tissue Res. 357 (2014) 515-526. https://doi.org/10.1007/s00441-014-1945-2.

[38] A. Hamadi, M. Bouali, M. Dontenwill, H. Stoeckel, K. Takeda, P. Ronde, Regulation of focal adhesion dynamics and disassembly by phosphorylation of FAK at tyrosine 397, J. Cell. Sci. 118 (2005) 4415-4425. https://doi.org/10.1242/jcs.02565.

[39] M. Bharadwaj, N. Strohmeyer, G.P. Colo, J. Helenius, N. Beerenwinkel, H.B. Schiller, R. Fassler, D.J. Muller,alphaV-class integrins exert dual roles on alpha5beta1 integrins to strengthen adhesion to fibronectin, Nat. Commun. 8 (2017) 14348. https://doi.org/10.1038/ncomms14348.

[40] A. Gonzalez-Pujana, E. Santos, G. Orive, J.L. Pedraz, R.M. Hernandez,Cell microencapsulation technology: Current vision of its therapeutic potential through the administration routes, Journal of Drug Delivery Science and Technology. (2017). https://doi.org/10.1016/j.jddst.2017.03.028.

[41] A. Mardilovich, J.A. Craig, M.Q. McCammon, A. Garg, E. Kokkoli,Design of a novel fibronectin-mimetic peptide-amphiphile for functionalized biomaterials, Langmuir. 22 (2006) 3259-3264. https://doi.org/10.1021/la052756n.

[42] A. Sharma, J.A. Askari, M.J. Humphries, E.Y. Jones, D.I. Stuart,Crystal structure of a heparin- and integrinbinding segment of human fibronectin, EMBO J. 18 (1999) 1468-1479. https://doi.org/10.1093/emboj/18.6.1468.

[43] R. Cruz-Acuna, A.J. Garcia,Synthetic hydrogels mimicking basement membrane matrices to promote cellmatrix interactions, Matrix Biol. 57-58 (2017) 324-333. https://doi.org/10.1016/j.matbio.2016.06.002. 
[44] S.G. Ricoult, A.S. Nezhad, M. Knapp-Mohammady, T.E. Kennedy, D. Juncker,Humidified microcontact printing of proteins: universal patterning of proteins on both low and high energy surfaces, Langmuir. 30 (2014) 1200212010. https://doi.org/10.1021/la502742r. 